\title{
An Image Searching Framework using Hybrid Algorithm
}

\author{
Bello B. O. \\ Federal Polytechnic \\ Ile-Oluji, Nigeria
}

\author{
Iwayemi A. \\ Federal Polytechnic \\ Ile-Oluji, Nigeria
}

\begin{abstract}
Searching the internet, World Wide Web or databases are only possible with textual index. In other words, images could only be retrieve by having searching parameter(s) that are in textual form. Also, text information is only retrievable by providing a textual search index. No matter how real or identical an image is to the real object, searching cannot be done through it. There will be need for a provision of a matching information in textual form if searching will be successful. This paper presents an image searching framework using facial recognition. The framework uses the hybrid of Genetic algorithm (GA) and Speed Up Robust Features (SURF) algorithm to locate face boundaries, extract facial features and interest points for recognition so as to retrieve a perfect match of image queried from the database. The whole process includes: the query interface, where user will be able to upload image for query and the next is detecting and extracting the facial features, the next step is to search the database for the best face matches.
\end{abstract}

\section{General Terms}

Image Recognition, Genetic Algorithm, SURF.

\section{Keywords}

Genetic Algorithm, SURF, Face detection, Face Extraction, Query, Pre-processing, Recognition, Extraction, Localization, Matching.

\section{INTRODUCTION}

Generally, Image searching is the act of retrieving information which are in lines with userse queries by searching through collections of images or simply database with the aid of different algorithms. [1] image search has become an important feature of multimedia, though [2] affirm that the actual way of image retrieval is the text based image retrieval technique (TBIR), but the techniques used in this work is image based and [3] numbers of research papers have been studied to find the current trends and approaches for image retrieval. The Facial recognition is an aspect of Computer Vision and also a method of biometric for identifying faces in an image or videos which involves detection and extraction of face boundaries and other facial features, other methods of biometric systems include; Speech Recognition, Fingerprint Recognition and so on. [4] Face recognition has recently gained important attention particularly throughout the past many years. The Image Searching using facial recognition system involves various steps and the three major steps includes; Detection of face boundaries in an image, Extraction of facial features detected from the face image and finally matched the features extracted together with those in the database. Example of this image searching include: Google of basic design having large database and relying on mixed retrieval for accuracy and the "Tineye image search engine". These search engines are dedicated to retrieving images by text or by search terms in the filename of the image and also from link text pointing to the image or text adjacent to the image for searching.

\section{LITERATURE REVIEW}

[5] used a fast and robust algorithm for the matching process yet it was dependent on only one algorithm. [6] provided efficient means for face detection, matching and annotation, working with unconstrained digital photos of variable quality, requiring no time-consuming training. Nevertheless, there were challenges on unconstrained images. [7] color based indexing was used as color feature of image and color compute though re-ranked and color texture only. In reporting a content based image indexing and retrieval, [8] calculate the distance between two images and ended up retrieving content of image only. [9] reported an approach of face detection using geometrical definition of human face, a method which was better than neural network identifying faces with variations in illumination. [10] utilizes eye and mouth center localization and also use geometrical information, thus, avoiding illumination problems and facial image intensity variations. [11] presented a means of indexing and retrieving photographic image though limited to geo-location and content. In their automatic extraction system, [12] presented a 3-D modelling of frontal facial features which is its scope whereas [13] specified facial localization. The design and implementation of an image search algorithm was presented by [14]. [15] presented a rapid object detection that brought together three distributed contributions and also minimized computation time while achieving high detection accuracy whereas [16] said after a coarse detection of facial region, face position parameters can be refined to a second phase.

\section{METHODOLOGY}

The method employed in this work follow a technological and logical processes, when user inputs intended image into the searching system, the system will then perform face recognition using series of modeling's and algorithm, to search through the trained face images stored in the database and retrieve the particular face image that matches the user input face image. And if there is no match for the face image inputted, the searching system will return a message describing the face image as unknown for the user. The algorithms used are further discussed in this section. Below are the stages involved in searching and recognizing face.

- Input image: Here image will be input and captured into the searching system.

- Detect Face Region in the image: Detecting the face is the very first step in image matching process. In this stage the very part of the digital image that contains human face is located. Here two techniques are employed, the key points for the image are located using the Speed-Up Robust Features (SURF) and later fed to the genetic algorithm for optimization.

- Extract Facial Features: The second stage of the matching system involves the extraction of the features detected and localized by the genetic algorithm in the first stage. In this stage, Speed-Up 
Robust Features (SURF) and Genetic Algorithm (GA) are used for the extraction of the facial features and optimization to adapt the face models. The facial features include the eye, lips, nostrils and chin contours.

- Compare features extracted with trained face image: In this stage the features will be compared with those image trained in the database.

- Display matched image: The trained face image that matched up with the input image will be retrieve and display to the screen with additional information of the face in the image such as Name, Age, Contact etc.

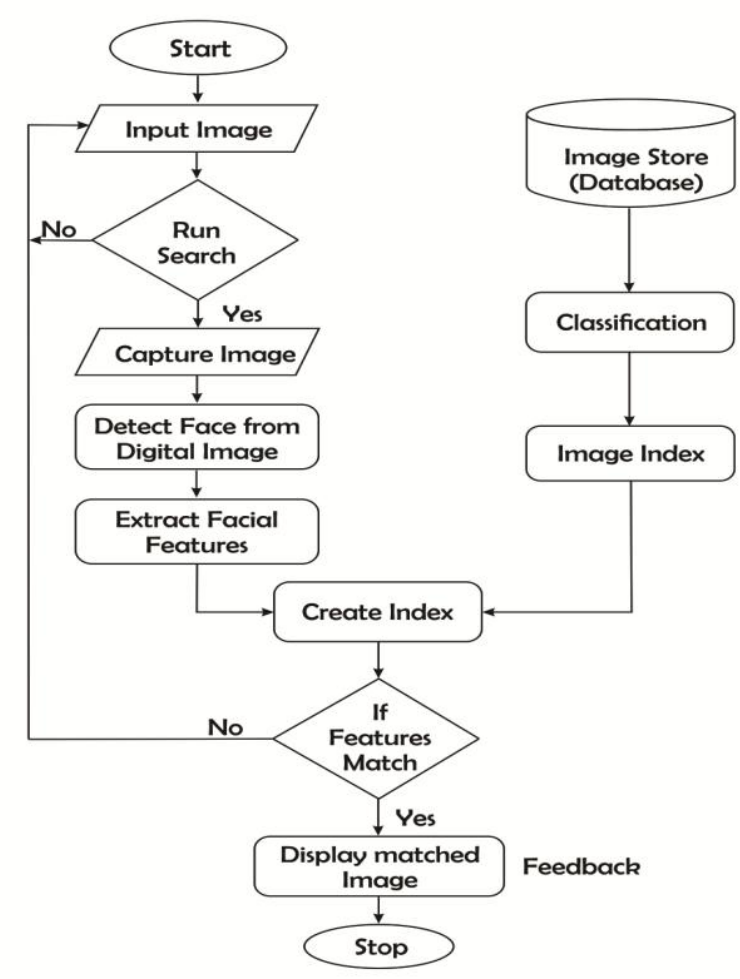

Fig 1: Image searching flow chart.

The image searching stages are represented by flow chart as shown in Figure 1.

\subsection{Genetic Algorithm (GA)}

Based on the concept of Darwin's theory of evolution, John Holland introduced Genetic Algorithm. The Genetic Algorithm is an unorthodox optimization problem built on the concept of natural genetic evolution of genes. Genetic algorithm is basically used for providing adequate solutions to search and optimization problems. The algorithm comprises of bio-inspired operator which include; Selection, Crossover and Mutation. While performing search with the genetic algorithm the problems solutions are firstly convert into chromosome made up of genes or symbols and sets of chromosome is regarded as population. For the genetic search to produce new generation below are the steps.

a. Evaluating the Fitness of the Chromosome At first the performance of each chromosome will to be evaluating according to their fitness i.e. to be in good solution. To determine how close, the solution is, each of the chromosomes is assigned a value according to the quality of surviving (fitness).

\section{b. Selection}

According to Darwinian evolution's theory, all chromosomes with high-quality fitness will be selected Selection occur after evaluating each of the chromosomes and the best among other are selected. Although there are many method of selection such as the Roulette-wheel and Rank selection, but the method of selection in this case is the Boltzmann Selection. The probability of visiting a point in optimization space $x \mathrm{j}$, is given by equation 1.0 .

$$
P(x i)=\frac{e^{-u(x i) I T}}{\sum_{i} e^{-u(x i) I T}}
$$

Where: The numerator contains the Boltzmann weighting term

The denominator is a normalization factor.

The Boltzmann selection procedure is represented by equation 2.0:

$$
F i(u(x))=e^{u i(x) I T}
$$

$\mathrm{U}, \mathrm{P}$, and $\mathrm{w}$ are define as in proportional selection. The formulations add a number of attractive features.

\section{c. Cross-over}

In this genetic operator, some genes of chromosome $\mathrm{A}$ and chromosome B regarded as parents are taken to generate a new chromosome regarded as the child. Figure 2 shows an example of two chromosome producing child.

Parent 1

Parent 2

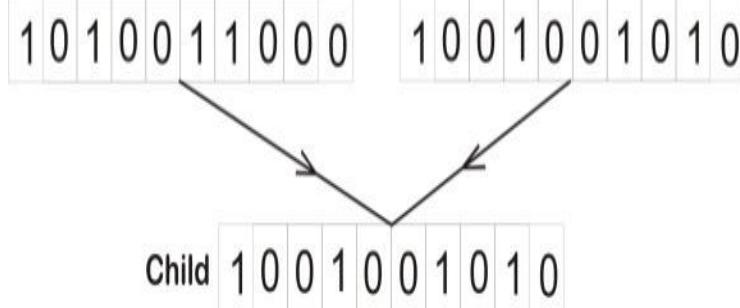

Fig 2: Crossover

d. Mutation

Mutation is another operator used in genetic search which can replace one or more genes presents in chromosome one example of mutation is the bit string mutation which works at random position. Figure 3 below shows an example of mutation.

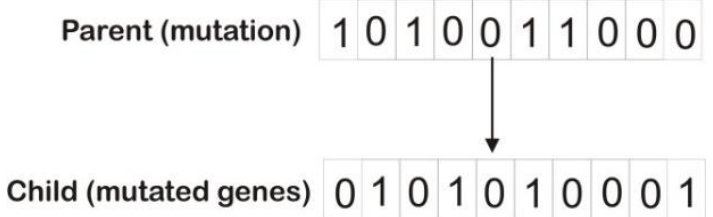

Fig 3: Mutation

\subsubsection{GA Algorithm}

The algorithm below summarized the Genetic Algorithm and represented with flow chart in figure 4 . 


\section{Algorithm}

1. Initialize the set of chromosomes (populations).

2. Evaluate the fitness of each set of chromosomes.

3. If the fitness of each set of chromosome meets the criteria, then jump to step 8 else continue with next step.

4. Select the chromosomes in order of fitness probability for mating.

5. Crossover selected parents.

6. Proceed with mutation

7. Proceed to step 2 (evaluating the fitness)

8. End

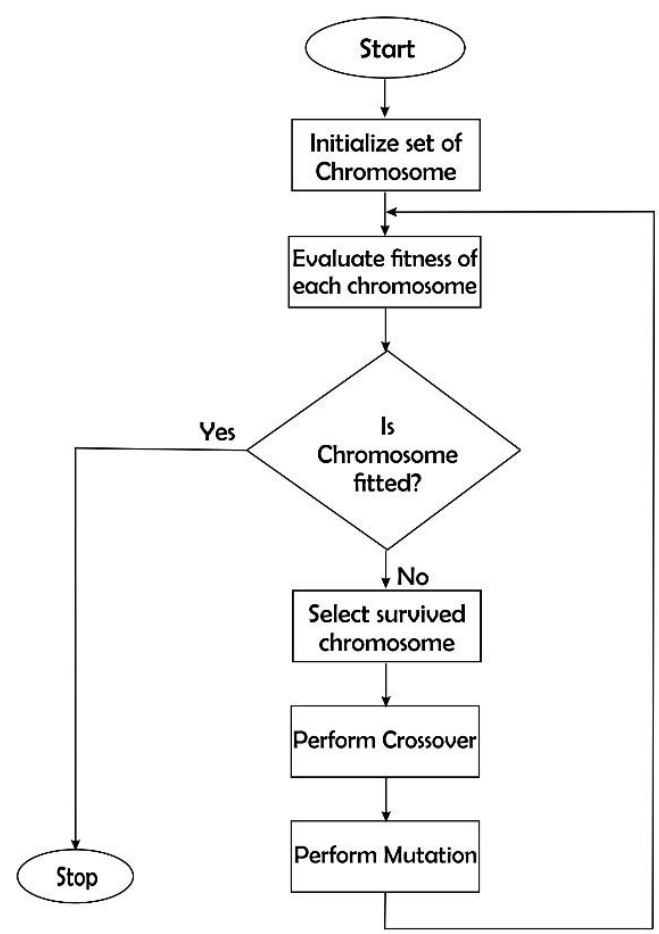

Fig 4: Genetic Algorithm flow chart

\subsection{Speed up Robust Features}

[17] Speed up Robust Features (SURF) is a scale and in-plane rotation invariant feature detection and descriptors. It comprises of interest points detector and descriptor. Although it was inspired by Scale Invariant Features Transform, is still much faster than the 128-dimensional local descriptors and also has the features of being computed efficiently. The SURF Detector locates the interest points in an image using the determinant of the approximate hessian matrix and the SURF Descriptor describes the features of the located interest points.

\subsection{Detecting Face from Input Image}

For the algorithm to work easily and faster, the image must undergo Pre-processing, normalization and histogram equalization. The Speed Up Robust Features detectors and descriptor locates the interest point and describes the interest points of the image.

For genetic algorithm to detect face in the image input the parameters below are considered and represented with a flow chart shown in Figure 7.

a. Original color image: This described the original image which is obviously a color image queried by user before any other action is performed on the image.

b. Preprocessing: In this step, the color image is first converted into gray scale image as shown in figure 5. After which the face, eyes, tip of the nose and corners of the mouth are all labeled, the points registered are used for normalizing each of the face to the same orientation, scale and also the position. So each of these normalized images are subjected to some image processing operations to in order to take into consideration different contrast and lighting conditions.Analysis: Gray scale image $=$ $(0.3 * \mathrm{R})+(0.59 * \mathrm{G})+(0.11 * \mathrm{~B})$

Where: $R$ represents RED and has contributed $30 \%$.

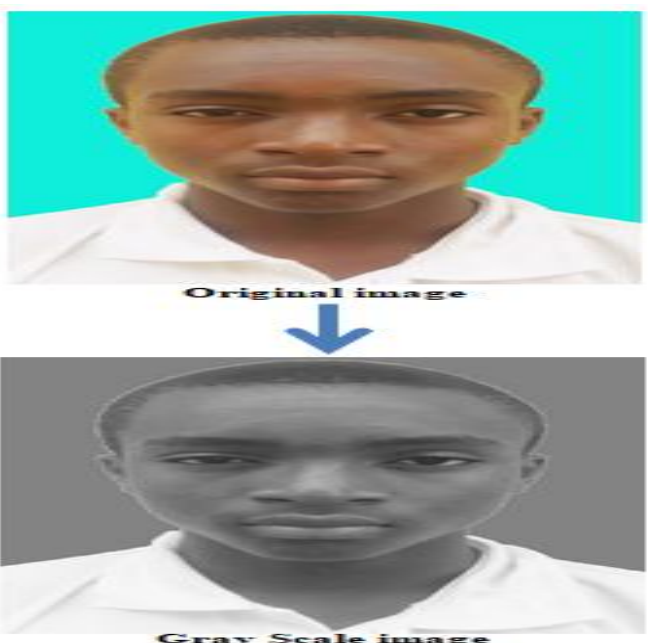

Fig 5: Original image converted to gray scale

$G$

represents GREEN and has contributed 59\% which is greater in all three colors. B represents BLUE and has contributed $11 \%$.

c. Image Enhancement: Histogram equalization is used to compensate for the lighting conditions and improve contrast of the image [18]. This is as a result of enhancing the image due to limitation in the lighting conditions that obviously may occur leading to poor contrast of the image.

Analysis: Let the histogram $\mathrm{h}(\mathrm{ri})=\mathrm{Pi} / \mathrm{n}$ of a digital face image consist of the color bins in the range [0, C-1],

Where: ri is the $i$-th color bin,

$P i$ is the number of pixels in the image with that color bin and $n$ is the total number of pixels in the image.

For any $r$ in the interval $[0,1]$, the cumulative sum of the bins provides with some scaling constant. The histogram equalization is performed by transforming the function $\mathrm{s}=$ $\mathrm{T}(\mathrm{r})$, which produces the mapping with the allowed range of pixel values, i.e., a level $\mathrm{s}$ for every pixel value $r$ in the original image and $0 \mathrm{~T}(\mathrm{r}) 1$ for $0 \mathrm{r} 1$, as shown in figure 6 . 


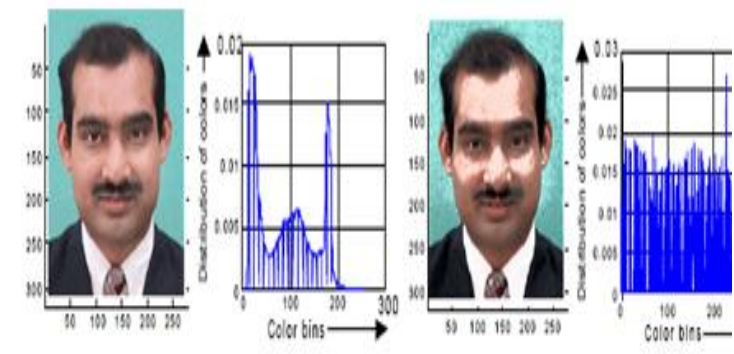

Fig 6: Histogram Equalization of face image. [19]

d. Filtering: due to some sources of noise that exit in the original input image the image is filtered, [19] Prewitt filter is used to suppress the noise.

e. Genetic Searching: The genetic searching is responsible for locating the face region, and searching for the facial features.

f. Fitness Function: The fitness value of the possible face region is computed by means of intensity similarity in order to verify if the region of the input image is a face.

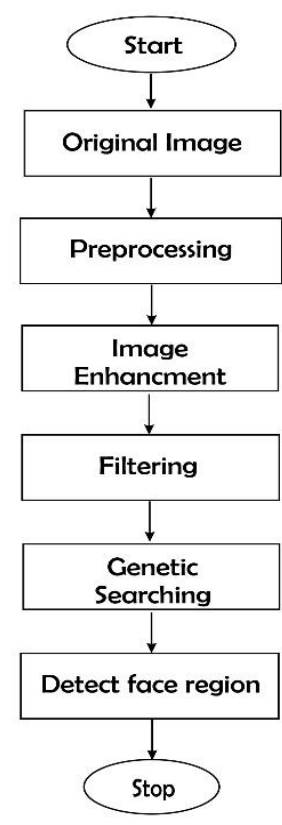

Fig 7: Fundamental steps for face detection

\subsection{Extracting Features}

The second stage of the matching system involves the extraction of the features and interest points detected and describe in the previous stage. In this stage, Speed-Up Robust Features (SURF) and Genetic Algorithm (GA) are used for the extraction of the facial features and optimization to adapt the face models. For the case of SURF algorithm features are extracted from the input image using the SURF detectors and descriptor by extracting interest points at first before the initial process such as pre-processing normalization and equalization of histogram. In genetic the facial features include the eye, lips, chin contours and nostrils. Symmetry property of human face was used for the estimation of the location of the eyes and mouth after which the deformable templates, the derivatives of [20] are employed to the estimated location guided by the genetic mating.
- The Symmetry Axis: [21] It is assumed that human face is symmetric in form and an axis of symmetry is found by a pixelwise scoring method with tilt adjustments limited to the range of $20^{\circ}$ as shown in Figure8. This method allows the non-facial background effects to be remove and the calculations are done on the lower resolution image excluding the image itself. Below is the step in finding the axis of symmetry.

To calculate the axis of symmetry, the ordinate of the center of symmetry is fixed at $y=Y / 2$. This axis is later shifted laterally and also rotates by small increments through the ranges of $\mathrm{X}$ and $\Theta$.

The image $\mathrm{I}(\mathrm{x}, \mathrm{y})$ with lower resolution and dimensions $\mathrm{X} * \mathrm{Y}$ is scanned between $\left[-20^{\circ}, 20^{\circ}\right]$ for integer values of $\Theta$. For every $(X, \Theta)$ parameter pair, a symmetry score is obtained using the equation 7.0 below For every $\left(\mathrm{x}_{0}\right.$ , $\left.\mathrm{y}_{\mathrm{o}}\right)$ point on the left hand side of the axis in this algorithm, the mirror image point, called $\left(\mathrm{x}_{\mathrm{s}}, \mathrm{y}_{\mathrm{s}}\right)$ is found with respect to the parameters $\left(\mathrm{X}_{\mathrm{k}}, \Theta_{\mathrm{k}}\right)$.

$\mathrm{N}$ is the total number of points that are used for calculating the symmetry measure which in general less than $\mathrm{X}$

Y. The mirror symmetry comparison is achieved with all the pixels for which a counterpart can be found within the borders of the image. The highest symmetry is represented by the smallest value of $S(X, \Theta)$, so that the symmetry axis is determined as the $\left(\mathrm{X}_{\mathrm{k}}, \Theta_{\mathrm{k}}\right)$ parameters that make $\mathrm{S}(\mathrm{X}, \Theta)$ function minimum. However, this approach is based on analysis of [21]. The equation is determined as shown in equation 3.0, 4.0, 5.0, 6.0 and 7 below.

$$
\begin{gathered}
x_{i}=\frac{x+\frac{X \tan \theta}{2}+\tan ^{2} \theta x_{o}-\tan \theta y_{o}}{1+\tan ^{2} \theta} \\
y_{i}=y_{o}-\tan \theta x_{o}+\tan \theta x_{i} \\
x_{s}=\left(x_{i}-x_{o}\right)+x_{i} \\
y_{s}=\left(y_{i}-y_{o}\right)+y_{i} \\
s(x, \theta)=\frac{1}{N} \sum_{y=\frac{Y}{4}}^{\frac{3 Y}{4}} \sum_{x=X=\frac{X}{O}}^{X+\frac{x}{o}}\left|I\left(x_{s}, y_{s}\right)-I\left(x_{o}, y_{o}\right)\right|
\end{gathered}
$$

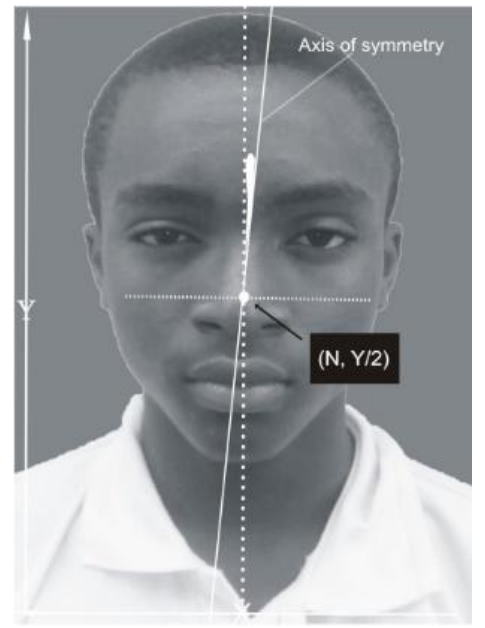

Fig 8: Axis of symmetry 
- $\quad$ Pre-Processing: After the completion of the previous symmetry axis calculation, a local maximum operation is then subjected to the image. The local maximum operation is defined as shown in equation.8.0.

$$
\left.I_{e}(x, y)=u(I(x, y))-M_{t}\right) \times 255
$$

Where: $I_{e}(x, y)$ is the output of the local maximum operator.

$M_{t}$ is the average of the window centered in the pixel, and $u$ is the unit step function.

Each of pixels is binarized to the value 255 if its gray value is above that of the window mean, and 0 shown in figure 9. Otherwise as shown in figure 10.

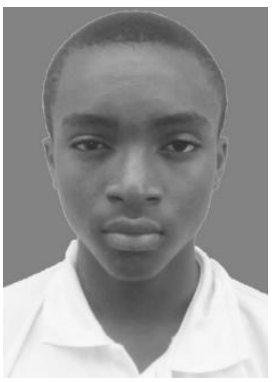

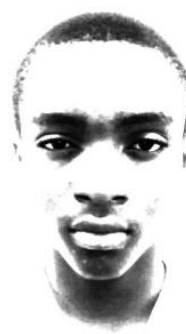

b
Fig 9: (a) Original image with axis of symmetry found, (b)binarised map.

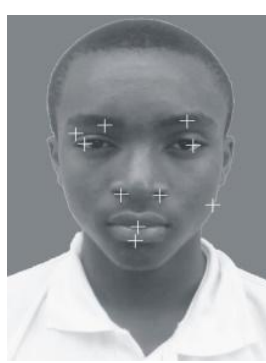

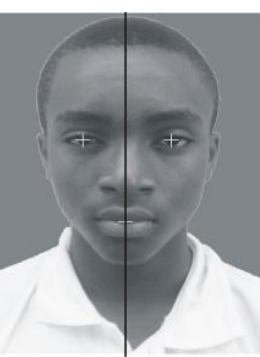

b
Fig 10: (a) The center of masses, (b) The eyes and mouth located

- Energy Field: Energy field was generated in order for the template fitting around the eyes and the contours of the lip to be introduced. The location of the eyes and mouth are estimated already, therefore rectangular frames are drawn on the estimated area for processing.

- Templates and Genetic Deformation: The deformable template adopted contains less parameter and is the simplified version of the one proposed by [20] because only the eye contours is located.

\subsection{Face Recognition}

This is the last stage of recognizing face. The facial features detected and extracted from the queried image are compared together with the features of each faces class store in the database. The search model is shown in Figure 11.

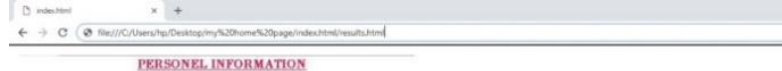

PERSONEL, INPORMATION

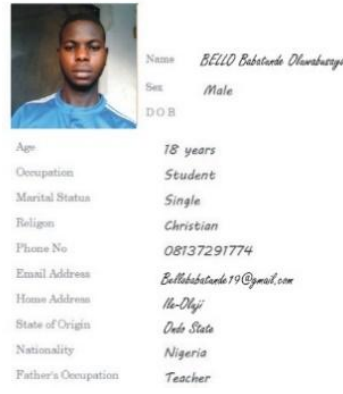

Fig 11: Model of the Image Searching.

\section{RESULT AND DISCUSSIONS}

Figure12. shows the interface of the image searching system where user can drop image to input into the system for querying by click the "upload" button and "Find Match" button to search for face in the database that match face of in the image input. Figure13 shows the information retrieved from the database. This technique was implemented in python programing language assisted with other packages and run under Intel(R) Core(TM) i5 CPU M 540 @ 2.53GHZ with 4.00GB RAM.

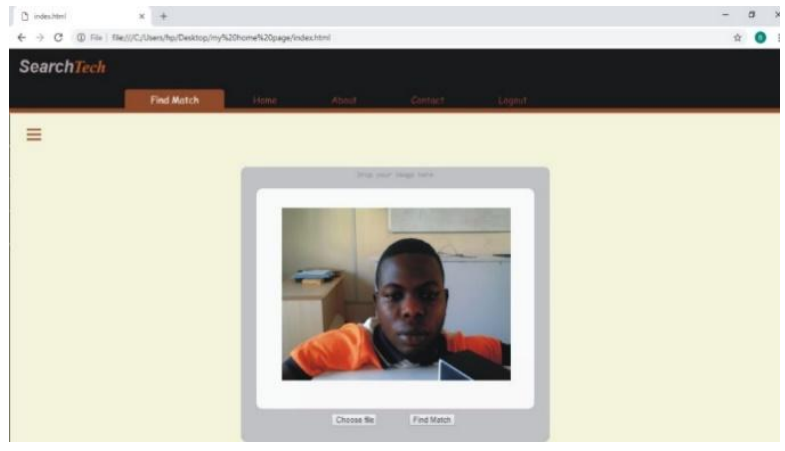

Fig 12: Searching interface.

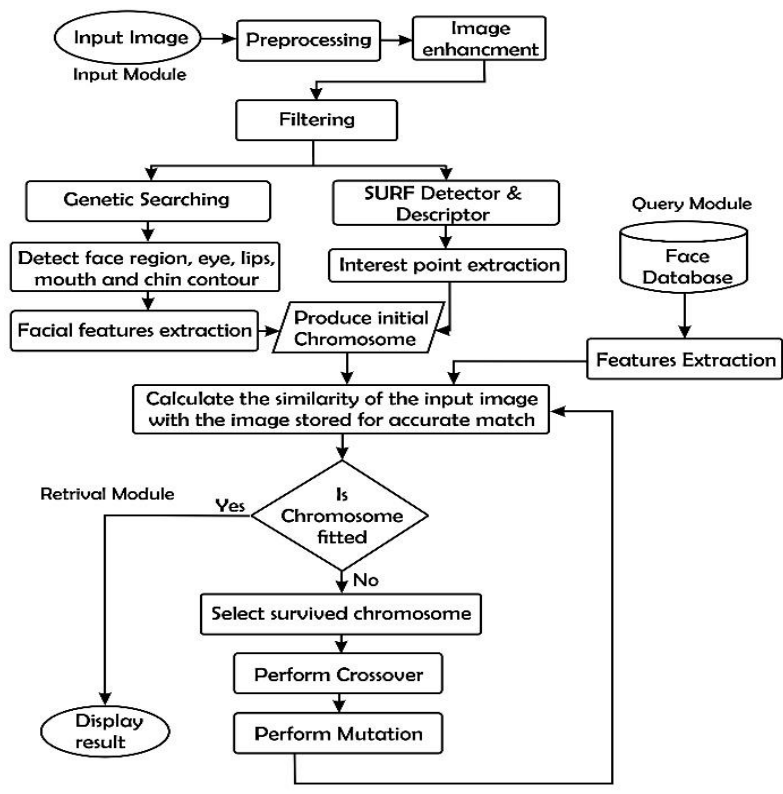

Fig 13: Image searching result. 


\section{CONCLUSION}

This work presented a genetic algorithm (GA) together with Speed-up Robust Features (SURF) to search for face image in database thereby recognizing an image. The genetic algorithm and Speed-up Robust Features method gives a suitable rate of detecting, extracting, and matching faces together more efficiently than some other methods. This work can still be improved using some other algorithm which are optimizable. From a technology perspective the image search engine requires large scale of image databases that will be connected together with the search engine. The image search engine will enable user to easily search for the details of the queried image.

\section{REFERENCES}

[1] Dipalee, N. and Sandeep, U. 2014. Survey on Methodologies Used for Web Image Search, International Journal of Computer Applications, Vol. 107, No. 18.

[2] Lixin, D., Wen, L., Ivor, W., and Dong, X. 2011. Improving Web Image Search by Bag-Based Re-ranking, IEEE Transactions On Image Processing, Vol. 20, No. 11.

[3] Jugal, K., Maria A. and Xiaobo, Z. 2005. Implementation of an Image Search Engine, M.S. Project Report Computer Science Dept. p. 4.

[4] Vijayarani and Vinupriya 2014. Facial Image Classification and Searching -A Survey, International Journal of Information Technology, Modelling and Computing, Vol. 2, No. 2.

[5] Unmesh, M. and Samir, K. 2016. Face matching using SURF feature points, International Journal of Research in Advanced Engineering and Technology, Vol. 2, No. 1, pp. 16-18.

[6] Eugene, B. and Szil'ard, V. 2016. FaceMatch: real-world face image retrieval, U.S. National Library of Medicine Lister Hill National Center for Biomedical Communications, Vol. 709, pp. 405-419.

[7] Mayuri, D., Joshi, R., Deshmukh, M., Kalashree, N., Hemke, Ashwini, B. and Rakhi, W. 2014. Image Retrieval and Re-Ranking Techniques - A Survey, International Journal (SIPIJ), Vol. 5, No. 2, pp. 1-14.

[8] Avinash, N. and Meshram, B. 2013. Content Based Image Indexing and Retrieval, International Journal of Graphics and Image Processing, Vol. 3, No. 4, pp. 235246.

[9] Tanmoy, M., Anupam, N., Ashok, D. and Muktinath, B. 2010. An Approach of Face Detection using Geometrical
Definition of Human Face, National Conference on Computational Instrumentation CSIO, Chandigarh, India, pp. 97-99.

[10] Stylianos, A., Nikos, N. and Ioannis, P. 2008. Facial Feature Detection using Distance Vector Fields, Department of Informatics, Vol. 42, No. 7, pp. 13881398.

[11] Yong-Hawan, L., Bonam, K. and Heung-Jun, K. 2012 Indexing and Retrieving Photographic Image Using a Combination of Geo-Location and Content Based Features, Computing and Informatics, Vol. 30, No. 6, pp. 1115-1129.

[12] Vladimir, V., Stanislav, S. and Anna, D. 2007. Automatic Extraction of Frontal Facial Features, Dept. of Computational Mathematics and Cybernetics, Moscow State University.

[13] Paola, C., Raffaella, L. and Giuseppe, L. 2007. Automatic Facial Feature Extraction for Face Recognition, University degli Studi di Milano Italy, pp. 3-58.

[14] Zhengxi, W., Pan, Z. and Liren, Z. 2014. Design and implementation of image search algorithm. America Journal of Software Engineering and Applications', Vol. 3, No. 6, pp. 90-94.

[15] Paul, V. and Michael, J. 2001. Rapid Object Detection using a Boosted Cascade of Simple Features, Institute of Electrical, Electronic Engineering, pp. 1-9.

[16] Oliver, J., Klaus, J. and Robert, W. 2001 Robust Face Detection Using the Hausdorff Distance, Biometric Person Authentication, Springer, Lecture Notes in Computer Science, Halmstad, Sweden, 2091, pp. 90-95.

[17] Geng, D., Fei, S. and Anni, C. 2016. Face Recognition using SURF Features, Pattern Recognition and Computer Vision

[18] Wang, J., Tan, T. 2000. A New Face Detection Method Based on Shape Information, Pattern Recognition. Lett. 21: pp. 463-471.

[19] Saifuddin, M., Rubayat, P., Liton, J., Al-Almin, B. 2007. Robust Face Detection using Genetic Algorithm, Information Technology Journal, Vol. 6, No. 1.

[20] Yuille, A., Hallinan, P. and Cohen 1989. Feature Extraction from Face using Deformable Templates, International Journal of Computer Vission, Vol. 8.

[21] Bilgin, E., Bulent, S. and Emin, A. 1996. Facial Feature Extraction using Genetic Algorithms, Bogazici University Electrical Engineering Department, Bebek, 80815, Istanbul. 